\title{
Prognostic value of computed tomography score in patients after extracorporeal cardiopulmonary resuscitation
}

\author{
Jeong-Am Ryu ${ }^{1,2+}$, Young Hwan Lee ${ }^{3 \dagger}$, Chi Ryang Chung ${ }^{1}$, Yang Hyun Cho ${ }^{4}$, Kiick Sung ${ }^{4}$, Kyeongman Jeon ${ }^{1,5}$, \\ Gee Young Suh ${ }^{1,5}$, Taek Kyu Park ${ }^{6}$, Joo Myung Lee ${ }^{6}$, Minjung Kathy Chae ${ }^{7}$, Jeong-Ho Hong ${ }^{8}$, Sei Hee Lee', \\ Hyoung Soo Kim ${ }^{10}$ and Jeong Hoon Yang ${ }^{1,6^{*}}$
}

\begin{abstract}
Background: We evaluated whether Alberta Stroke Program Early Computed Tomography Score (ASPECTS) with some modifications could be used to predict neurological outcomes in patients after extracorporeal cardiopulmonary resuscitation (ECPR).

Methods: This was a retrospective, multicenter, observational study of adult unconscious patients who were evaluated by brain computed tomography (CT) within 48 hours after ECPR between May 2010 and December 2016. ASPECTS, bilateral ASPECTS (ASPECTS-b), and modified ASPECTS (mASPECTS) were assessed by ROC curves to predict neurological outcomes. The primary outcome was neurological status upon hospital discharge assessed with the Cerebral Performance Categories (CPC) scale.

Results: Among 58 unconscious patients, survival to discharge was identified in 25 (43.1\%) patients. Of these 25 survivors, 19 (32.8\%) had good neurological outcomes (CPC score of 1 or 2). Interrater reliability of CT scores was excellent. Intraclass correlation coefficients of ASPECTS, ASPECTS-b, and mASPECTS were 0.918 ( $95 \% \mathrm{Cl}, 0.865-0.950)$, $0.918(95 \% \mathrm{Cl}, 0.866-0.951)$, and 0.915 (95\% Cl, 0.860-0.949), respectively. The predictive performance of mASPECTS for poor neurological outcome was better than that of ASPECTS or ASPECTS-b (C-statistic for mASPECTS vs. ASPECTS, 0.922 vs. $0.812, p=0.004$; mASPECTS vs. ASPECTS-b, 0.922 vs. $0.818, p=0.003$ ). A cutoff of 25 for poor neurological outcome had a sensitivity of $84.6 \%(95 \% \mathrm{Cl}, 69.5-94.1 \%)$ and a specificity of $89.5 \%(95 \% \mathrm{Cl}, 66.9-98.7 \%)$ in mASPECTS.
\end{abstract}

Conclusions: mASPECTS might be useful for predicting neurological outcomes in patients after ECPR.

Keywords: Brain computed tomography, Cardiopulmonary resuscitation, Extracorporeal membrane oxygenation

\section{Background}

Neurological outcome is an important issue in patients who survive cardiac arrest. In these survivors, several predictors of neurological outcomes, such as physical examination, several biomarkers, and electrophysiologic studies, have been reported [1,2]. Brain imaging might also be helpful for predicting neurological outcomes after

\footnotetext{
*Correspondence: jhysmc@gmail.com

${ }^{\dagger}$ Jeong-Am Ryu and Young Hwan Lee contributed equally to this work. 'Department of Critical Care Medicine, Samsung Medical Center,

Sungkyunkwan University School of Medicine, 81 Irwon-ro, Gangnam-gu, Seoul 06351, Republic of Korea

${ }^{6}$ Division of Cardiology, Department of Medicine, Samsung Medical Center, Sungkyunkwan University School of Medicine, Seoul, Republic of Korea Full list of author information is available at the end of the article
}

cardiac arrest $[3,4]$. Acute hypoxic-ischemic injury might be manifested by loss of gray-white matter discrimination, brain swelling, or low-density lesions on brain computed tomography (CT) $[5,6]$. However, these changes in brain CT are likely to be subjective and difficult to quantify [7]. The Alberta Stroke Program Early Computed Tomography Score (ASPECTS) is a widely used screening tool that provides a framework for quantifying the extent of ischemic hypodensity or hypoattenuation in the middle cerebral arterial (MCA) territory [8]. In the setting of conventional cardiopulmonary resuscitation (CPR), ASPECTS with some modifications has been found to be useful to predict neurological outcomes of post-cardiac arrest patients $[9,10]$. However, whether ASPECTS might be helpful to

(c) The Author(s). 2018 Open Access This article is distributed under the terms of the Creative Commons Attribution 4.0 International License (http://creativecommons.org/licenses/by/4.0/), which permits unrestricted use, distribution, and 
systemically estimate neurological outcomes of survivors after extracorporeal cardiopulmonary resuscitation (ECPR) has not been reported. Therefore, the objective of this study was to investigate whether ASPECTS with some modifications could be used to predict neurological outcomes of patients after ECPR.

\section{Methods}

\section{Study population}

This was a retrospective, multicenter, observational study of adult patients who underwent ECPR during hospitalization at Hallym University Medical Center (HUMC) and Samsung Medical Center (SMC) between May 2010 and December 2016. This study was approved by the institutional review boards of HUMC (HUMC 2015i128) and SMC (SMC 2017-11-088-002). The requirement for informed consent was waived owing to the study's retrospective nature. Clinical and laboratory data were collected by a trained study coordinator using a standardized case report form. We included patients who underwent ECPR during the study period. Those who were unconscious (a score $<9$ on the Glasgow
Coma Scale) [7] upon admission to the hospital after cardiac arrest and those who had a brain CT scan within 48 hours after ECPR were selected. Of these patients, we excluded patients who were under 18 years of age; those who had malignancy whose expected lifespan was less than 1 year; those who had insufficient medical records; and those who had a history of head trauma, neurosurgery, or chronic neurological abnormality upon intensive care unit (ICU) admission. In addition, we excluded patients who had low ASPECTS and were suspected to have brain lesions due to factors other than hypoxic ischemic encephalopathy. We excluded patients for whom we could not define neurological status because of continuous sedation or death of unknown causes or causes other than brain death. Finally, a total of 58 patients with cardiac arrest who were rescued by venoarterial extracorporeal membrane oxygenation (ECMO) were analyzed in this study (Fig. 1).

\section{Definitions and outcomes}

In this study, ECPR was defined as both successful venoarterial ECMO implantation and pump-on with

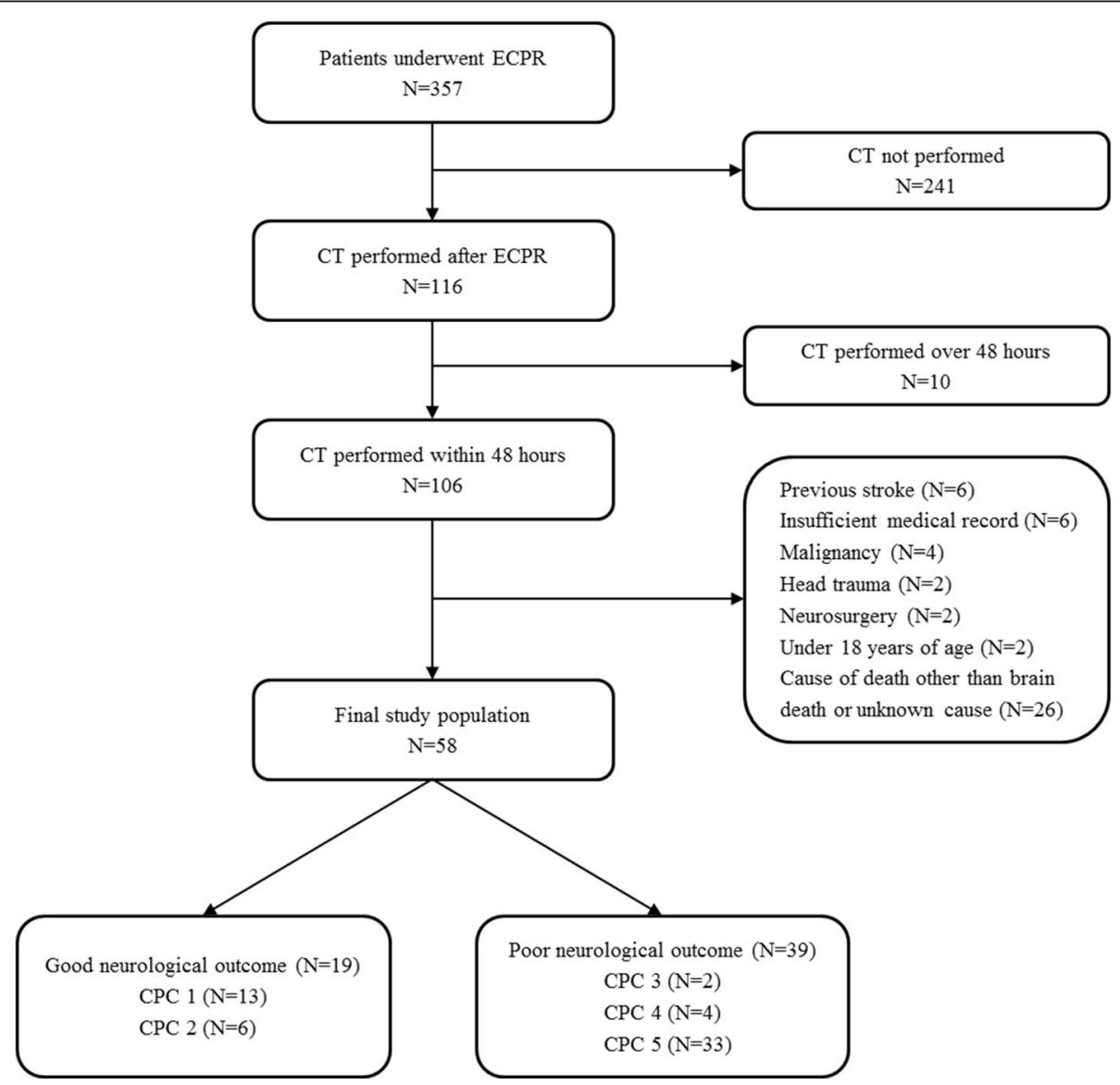

Fig. 1 Study flowchart. ECMO Extracorporeal membrane oxygenation, CPR Cardiopulmonary resuscitation, ECPR Extracorporeal cardiopulmonary resuscitation, CPC Cerebral Performance Categories scale 
cardiac massage during index procedure in patients with cardiac arrest. When a return of spontaneous circulation (ROSC) occurred during ECMO cannulation, practitioners typically did not remove the cannula or stop the ECMO pump-on process $[11,12]$. ECMO pump-on was defined by stopping chest compressions following successful ECMO implantation and activation. ECMO flow was then gradually increased until the patient was hemodynamically stable. The resuscitation procedure was performed in the same way as described in our previous study $[7,13]$. Arrest to ECMO pump-on time was defined as the time from cardiac arrest to the time at which the ECMO pump was turned on. Targeted temperature management was performed with surface cooling devices. We used a commercial temperature regulation system consisting of a hydrogel pad (Arctic Sun ${ }^{\circ}$; Medivance Corp., Louisville, CO, USA) or a cooling blanket. Surface cooling and the targeted temperature were determined by each intensivist in the ICU according to the therapeutic hypothermia protocol [14]. The primary outcome was neurological status upon hospital discharge assessed with the Glasgow-Pittsburgh Cerebral Performance Categories (CPC) scale (1 to 5) [15]. CPC scores of 1 and 2 were classified as good neurological outcomes. CPC scores of 3, 4, and 5 were considered as poor neurological outcomes. We thoroughly reviewed medical records. Patients were graded on the CPC scale by two independent neurologists. Brain death was confirmed by neurologists. Brain death was diagnosed on the basis of absence of brainstem reflex by neurologic examination, electroencephalography (EEG), and apnea test. Sometimes, transcranial Doppler or brain CT angiography was needed to diagnose brain death when apnea test was impossible due to extracorporeal circulation. Prognostication upon electrocerebral inactivity on EEG and apnea led to termination of therapy or consideration of organ transplant. All recorded brain CT scans were taken within 48 hours after ROSC in this study. After successful ECPR, for patients who had a rapid recovery of mental status and neurological deficits, brain $\mathrm{CT}$ was not performed. Otherwise, brain $\mathrm{CT}$ was performed to determine whether control of increased intracranial pressure was needed. Brain CT was also used to exclude intracranial hemorrhage before therapeutic hypothermia by the intensivist. For all CT studies, 64-channel scanners (at HUMC, SOMATOM Sensation; Siemens, Erlangen, Germany; at SMC, Light Speed VCT; GE Healthcare, Milwaukee, WI, USA) with a 5-mm slice width were used. Brain CT images were reviewed by two independent neurologists. Investigators who were blinded to clinical information opened these CT scans for each patient using commercial image-viewing software (at HUMC, PiView STAR; INFINITT Healthcare, Seoul, South Korea; at SMC, Centricity RA1000 PACS
Viewer; GE Healthcare, Milwaukee, WI, USA). To evaluate the extent of hypoxic-ischemic insult quantitatively, newly modified CT scores were used that are based on the original ASPECTS protocol [8]. The original ASPECTS was used to predict neurological outcomes in this study. Of original ASPECTSs for both hemispheres, the smaller value was used for analysis. To evaluate brain injuries in both hemispheres, bilateral ASPECTS (ASPECTS-b) was extended to both sides based on the original ASPECTS protocol [10]. In addition, modified ASPECTS (mASPECTS) was used in this study. Similar to ASPECTS-b, each MCA territory was scored with $0-10$ points. Anterior cerebral artery (ACA) and posterior cerebral artery (PCA) territories had 2 points corresponding to upper and lower levels of each territory. The brainstem and each cerebellar hemisphere had 1 point (Table 1, Fig. 2) [6]. Whenever there were early ischemic findings on CT images such as parenchymal hypoattenuation, cortical swelling with sulcal effacement, and loss of gray-white matter differentiation from each area, new CT scores were calculated by subtracting 1 point from the maximum of 20 (ASPECTS-b) or 31 (mASPECTS) $[6,10]$. A brain with diffuse infarction that involved all areas was scored zero.

\section{Resuscitation procedure}

CPR was led by the hospital's CPR team, and all data related to the CPR scene were recorded by a bedside nurse according to the Utstein-style guidelines [16]. The on-call ECMO team leader was called when CPR was performed for longer than 10 minutes or in the event of unstable vital signs or recurrent cardiac arrest. The ECMO team leader along with the CPR leader assessed the patient and decided whether to institute ECPR. ECPR was performed when a witnessed arrest was confirmed, the arrest persisted despite at least 10 minutes of conventional CPR, and the underlying cause of the arrest was considered reversible [17]. Cases in which ECPR was deferred included a short life expectancy ( $<6$ months), terminal malignancy, an unwitnessed collapse, limited physical activity, an unprotected airway, or CPR undertaken for longer than 60 minutes at the time of initial contact. Age alone did not constitute a contraindication for ECPR [17]. The ECMO team at our institution consists of cardiologists, cardiovascular surgeons, intensivists, specialized nurses, and perfusionists. Either the Capiox Emergency Bypass System (Terumo, Tokyo, Japan) or the Prolonged Life Support System (Maquet Cardiopulmonary, Hirrlingen, Germany) was used in all cases. A crystalloid solution such as normal saline or balanced solution was used for priming; no patient in this study underwent blood-primed ECMO. A percutaneous vascular approach was tried initially in all cases using the Seldinger technique; if percutaneous cannulation failed, a surgical cut-down exposure was performed [17]. The femoral vessels were the most 
Table 1 Scoring system of modified ASPECTS

\begin{tabular}{llll}
\hline & \multicolumn{2}{l}{ Side } & Scoring \\
\cline { 2 - 3 } Vascular territories & Right & Left & \\
\hline ACA lower (A1) & 1 & 1 & 2 \\
ACA upper (A2) & 1 & 1 & 2 \\
MCA (ASPECTS and ASPECTS-b) & & & 20 \\
$\quad$ MCA as per ASPECTS (C, caudate; & $0-10$ & $0-10$ & \\
$\quad$ I, insula; L, lentiform nucleus; & & & \\
IC, internal capsule; MCA, M1-M6) & & & \\
PCA lower (P1) & 1 & 1 & 2 \\
PCA upper (P2) & 1 & 1 & 2 \\
Cerebellum (CL) & 1 & 1 & 2 \\
Brainstem (B) & 1 & & 1 \\
Total score & & & 31 \\
\hline
\end{tabular}

Abbreviations: ACA Anterior cerebral artery, ASPECTS Alberta Stroke Program Early CT Score, MCA Middle cerebral artery, PCA Posterior cerebral artery

common sites of vascular access, and 14- to 17-French arterial cannulas and 20- to 24-French venous cannulas were placed [12]. Cardiac compression was stopped once ECMO initiation was deemed successful. Anticoagulation was accomplished with a bolus injection of unfractionated heparin followed by a continuous intravenous heparin infusion to maintain an activated clotting time between 150 and 180 seconds. The initial number of revolutions per minute of the ECMO device was adjusted to achieve an ideal cardiac index greater than $2.2 \mathrm{~L} / \mathrm{min} / \mathrm{m}^{2}$ of body surface area, central mixed venous oxygen saturation above $70 \%$, and a mean arterial pressure above $65 \mathrm{mmHg}$ [12]. Blood pressure was monitored continuously through an arterial catheter, and an artery in the right arm was used for arterial blood gas analysis to estimate cerebral oxygenation. After ECMO was established, the necessary steps were taken to treat the underlying cause of cardiac arrest, including percutaneous coronary intervention, coronary artery bypass grafting, heart transplant, and noncardiopulmonary surgery [12].

\section{Statistical analyses}

All data are presented as median and interquartile range (IQR) for continuous variables and number (percent) for categorical variables. Data were compared using the Mann-Whitney $U$ test for continuous variables and the chi-square test or Fisher's exact test for categorical variables. The predictive performance of each brain CT marker was assessed using AUC of the ROC curve for sensitivity vs. 1 - specificity. AUCs were compared using the nonparametric approach published by DeLong et al. [18] for two correlated AUCs. The optimal cut-off value of each brain CT marker for predicting poor neurological outcome was obtained by the ROC curve and Youden index $[19,20]$. Intraclass correlation coefficient (ICC) was used to analyze interrater reliability of ASPECTS-b and mASPECTS [21]. ICC was estimated using a single-measurement absolute-agreement and two-way mixed-effects model [21]. All tests were two-sided, and $p$ values $<0.05$ were considered statistically
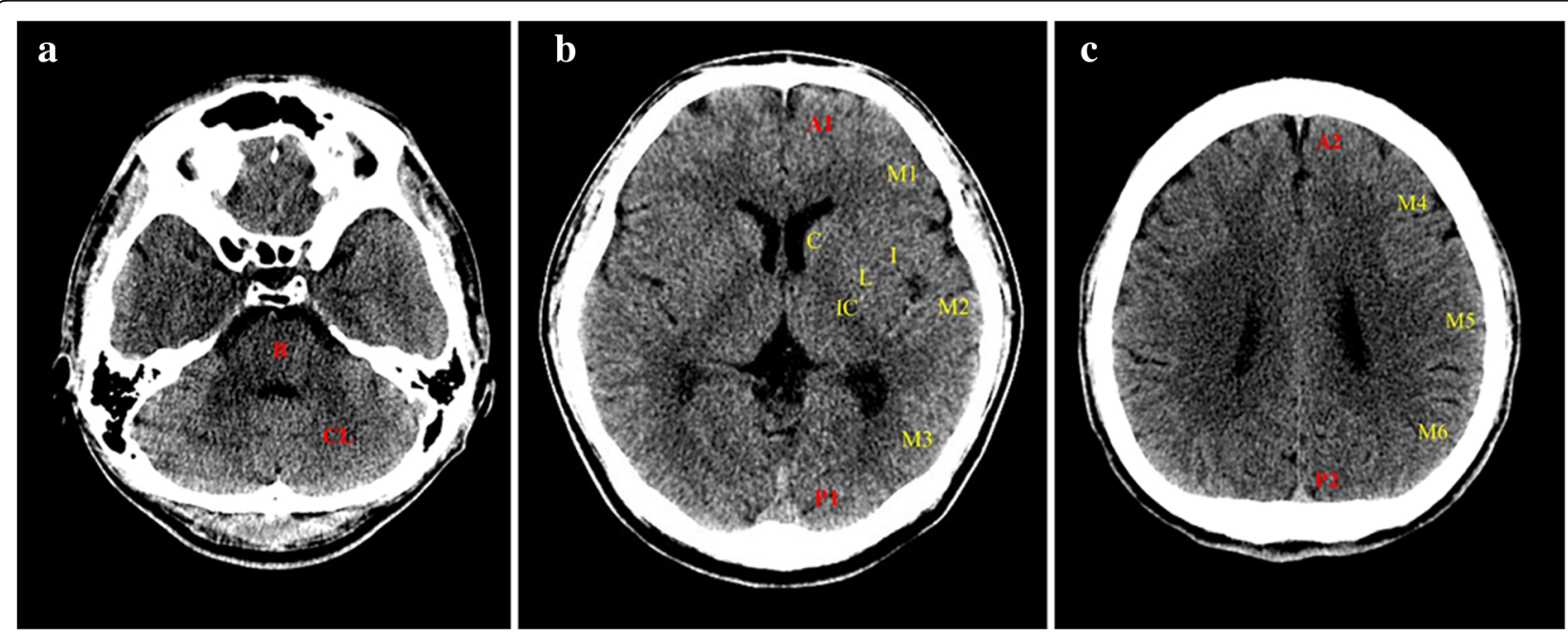

Fig. 2 Axial computed tomographic images at three levels (a, b, c). Modified Alberta Stroke Program Early Computed Tomography Score (ASPECTS) was used for analysis of images. The score was used to interpret the whole brain. It was not limited to specific slices. See Table 1 for further details. Bilateral yellow regions indicate ASPECTS-b (extended to both sides from the original ASPECTS protocol). Combined bilateral regions of yellow and red indicate modified ASPECTS. A1 Anterior cerebral artery (ACA) lower, A2 ACA upper, ASPECTS- $b$ Bilateral ASPECTS, B Brainstem, C Caudate, CL Cerebellum, I Insula, IC Internal capsule, L Lentiform nucleus, M1-M6 Middle cerebral artery territories, mASPECTS Modified ASPECTS, P1 Posterior cerebral artery (PCA) lower, P2 PCA upper 
significant. Data were analyzed using IBM SPSS statistics version 20 software (IBM, Armonk, NY, USA).

\section{Results}

\section{Baseline characteristics and clinical outcomes}

The median age of patients was 52 years (IQR, 37-58 years). Of 58 patients, 44 (75.9\%) were males. A cardiac cause of arrest was verified in 49 (84.5\%) patients. Thirty-one (53.4\%) patients experienced cardiac arrest in the hospital, and 27 (46.6\%) patients had cardiac arrest in an out-of-hospital setting. The median time from arrest to ECMO pump-on was 47.5 minutes (IQR, 29.0-63.0 minutes). Targeted temperature management was performed in $40(69.0 \%)$ patients using surface cooling devices. Only one patient had ROSC during cannulation. Baseline characteristics of ECPR patients are presented in Table 2. Characteristics of cardiac arrest are shown in Table 3. There were no significant differences in baseline or arrest-related characteristics between the two neurological outcome groups. Successful ECMO weaning was achieved in 35 (60.3\%) patients. Survival to discharge was identified in 25 (43.1\%) patients. Of these 25 survivors, 19 (32.8\%) had good neurological outcomes (CPC score of 1 or 2) (Fig. 1).

\section{Brain CT findings}

In this study, interrater reliabilities of CT scores were excellent. ICCs of ASPECTS, ASPECTS-b, and mASPECTS were 0.918 (95\% CI, 0.865-0.950), 0.918 (95\% $\mathrm{CI}, 0.866-0.951$ ), and 0.915 (95\% CI, 0.860-0.949), respectively. CT scores of ASPECTS, ASPECTS-b, and mASPECTS in the poor neurological outcome group were significantly lower than those in the good neurological outcome group (all $p<0.001$ ) (Table 4, Fig. 3a). In ROC curve analysis for the prediction of poor neurological outcome, the C-statistic of ASPECTS was 0.812 (95\% CI, 0.688-0.902). A cutoff $\leq 3$ had a sensitivity of $59.0 \%(95 \% \mathrm{CI}, 42.1-74.4 \%)$ and a specificity of $100 \%$ (95\% CI, 82.4-100\%). The C-statistic of ASPECTS-b was 0.818 (95\% CI, 0.694-0.907). A cutoff $\leq 14$ had a sensitivity of $66.7 \%(95 \% \mathrm{CI}, 49.8-80.9 \%)$ and a specificity of 94.7\% (95\% CI, 74.0-99.9\%). The C-statistic of mASPECTS was 0.922 (95\% CI, 0.821-0.976). A cutoff $\leq 25$ had a sensitivity of $84.6 \%$ (95\% CI, 69.5-94.1\%) and a specificity of $89.5 \%$ (95\% CI, 66.9-98.7\%). The predictive performance of mASPECTS for poor neurological outcome was better than that of ASPECTS or ASPECTS-b $(p=0.004$ and $p=0.003$, respectively). Among three regions (cortex, subcortex/basal ganglia, and brainstem/

Table 2 Baseline characteristics of patients

\begin{tabular}{|c|c|c|c|}
\hline & Good neurological outcome $(n=19)$ & Poor neurological outcome $(n=39)$ & $p$ Value \\
\hline Age, yr, median (IQR) & $50.0(32.0-54.5)$ & $54.0(45.0-61.0)$ & 0.136 \\
\hline Gender, male, $n$ (\%) & $15(78.9)$ & $29(74.4)$ & 0.955 \\
\hline Body mass index, $\mathrm{kg} / \mathrm{m}^{2}$ & $23.1(21.6-25.8)$ & $25.5(22.4-28.3)$ & 0.064 \\
\hline \multicolumn{4}{|l|}{ Medical history, $n$ (\%) } \\
\hline Diabetes mellitus & $3(15.8)$ & $15(38.5)$ & 0.147 \\
\hline Hypertension & $6(31.6)$ & $18(46.2)$ & 0.439 \\
\hline Malignancy & $1(5.3)$ & $5(12.8)$ & 0.669 \\
\hline Dyslipidemia & $1(5.3)$ & $6(15.4)$ & 0.496 \\
\hline Current smoker & $6(31.6)$ & $14(35.9)$ & 0.976 \\
\hline Previous myocardial infarction & $0(0)$ & $6(15.4)$ & 0.178 \\
\hline Targeted temperature management, $n(\%)$ & & & 0.928 \\
\hline Arctic Sun ${ }^{\circledast}$ & $11(57.9)$ & $24(61.5)$ & \\
\hline Cooling pad & $2(10.5)$ & $3(7.7)$ & \\
\hline Initial Glasgow Coma Scale score, median (IQR) & $3.0(3.0-3.0)$ & $3.0(3.0-3.0)$ & 0.208 \\
\hline \multicolumn{4}{|l|}{ Laboratory data on admission } \\
\hline Initial lactate, $\mathrm{mmol} / \mathrm{L}$ & $12.4(10.1-15.1)$ & $8.8(7.3-13.3)$ & 0.071 \\
\hline Hemoglobin before $\mathrm{ECMO}, \mathrm{g} / \mathrm{dl}$ & $15.0(12.6-15.5)$ & $13.2(11.3-14.8)$ & 0.099 \\
\hline Hemoglobin after ECMO, g/dl & $11.1(9.8-12.4)$ & $12.1(9.7-13.1)$ & 0.449 \\
\hline Total bilirubin, mg/dl & $0.7(0.3-0.9)$ & $0.8(0.5-1.1)$ & 0.210 \\
\hline Blood urea nitrogen, $\mathrm{mg} / \mathrm{dl}$ & $15.0(10.6-18.0)$ & $15.5(13.0-23.5)$ & 0.223 \\
\hline Creatinine, mg/dl & $1.1(1.0-1.3)$ & $1.2(1.0-1.7)$ & 0.292 \\
\hline Bicarbonate, IU/L & $11.7(10.3-17.9)$ & $17.7(13.2-20.9)$ & 0.121 \\
\hline
\end{tabular}

IQR Interquartile range, ECMO Extracorporeal membrane oxygenation 
Table 3 Characteristics of patients with cardiac arrest and procedure

\begin{tabular}{|c|c|c|c|}
\hline & Good neurological outcome $(n=19)$ & Poor neurological outcome $(n=39)$ & $p$ Value \\
\hline Type of cardiac arrest, $n(\%)$ & & & 0.451 \\
\hline Out-of-hospital cardiac arrest & $7(36.8)$ & $20(51.3)$ & \\
\hline In-hospital cardiac arrest & $12(63.2)$ & $19(48.7)$ & \\
\hline Bystander-witnessed cardiac arrest, $n(\%)$ & $19(100.0)$ & $35(89.7)$ & 0.371 \\
\hline Bystander-performed CPR, $n(\%)$ & $15(78.9)$ & $30(76.9)$ & 0.999 \\
\hline First monitored rhythm, $n$ (\%) & & & 0.474 \\
\hline Asystole & $2(11.8)$ & $10(26.3)$ & \\
\hline Pulseless electrical activity & $6(35.3)$ & $12(31.6)$ & \\
\hline Shockable rhythm (VT or VF) & $9(52.9)$ & $16(42.1)$ & \\
\hline Defibrillation, $n(\%)$ & $11(61.1)$ & $18(47.4)$ & 0.500 \\
\hline Arrest to ECMO pump-on time, min, median (IQR) & $47.0(19.0-55.0)$ & $48.0(34.5-65.0)$ & 0.359 \\
\hline Location of ECMO insertion, $n(\%)$ & & & 0.594 \\
\hline Intensive care unit & $3(15.8)$ & $7(17.9)$ & \\
\hline Catheterization room & $7(36.8)$ & $19(48.7)$ & \\
\hline Emergency room & $9(47.4)$ & $12(30.8)$ & \\
\hline Other & $0(0)$ & $1(2.6)$ & \\
\hline Cardiac cause of arrest, $n(\%)$ & & & 0.156 \\
\hline Ischemic & $6(37.5)$ & $21(63.6)$ & \\
\hline Nonischemic & $10(62.5)$ & $12(36.4)$ & \\
\hline
\end{tabular}

Abbreviations: CPR Cardiopulmonary resuscitation, VT Ventricular tachycardia, VF Ventricular fibrillation, ECMO Extracorporeal membrane oxygenation, IQR Interquartile range

cerebellum) for mASPECTS, the score of cortex had the best predictive performance for poor neurological outcome after ECPR (Fig. 3b).

There were only ten patients for whom follow-up CT scans were performed within 7 days (three good neurological outcomes and seven poor neurological outcomes) during ECMO support. Four patients had lower CT scores than previous scans, and another six patients had the same scores as for previous scans. All four patients who had lower CT scores than on previous scans had poor neurological outcomes.

Portable EEG was performed in 30 patients within 7 days after ECPR. Continuous slow (36.7\%) and background suppression (23.3\%) were the most common EEG findings. One-half of patients received continuous intravenous sedatives during EEG monitoring. Therefore, EEG findings might have reflected the use of sedatives as well as hypoxic brain injury. All patients who had well-known malignant EEG patterns such as electrocerebral inactivity, burst suppression, or generalized periodic epileptiform discharges had poor neurological outcomes. The EEG findings are shown in Additional file 1.

\section{Discussion}

In the present study, we evaluated whether ASPECTS with some modifications could be used to predict

Table 4 mASPECTS and ASPECTS-b according to neurological outcomes

\begin{tabular}{llll}
\hline & Good neurological outcome $(n=19)$ & Poor neurological outcome $(n=39)$ & $p$ Value \\
\hline ASPECTS (range 0-10) & $9.0(7.5-10.0)$ & $1.0(0-8.0)$ & $<0.001$ \\
${\text { ASPECTS- } b^{\text {a }} \text { (range 0-20) }}$ (19.0 (16.0-20.0) & $6.0(0-17.5)$ & $<0.001$ \\
mASPECTS $^{\text {b }}$ (range 0-31) & $29.0(27.0-31.0)$ & $9.0(0-25.0)$ & $<0.001$ \\
Cortex (range 0-20) & $20.0(18.5-20.0)$ & $4.0(0-14.0)$ & $<0.001$ \\
Subcortex/basal ganglia (range 0-8) & $8.0(6.0-8.0)$ & $0(0-8.0)$ & 0.001 \\
Brainstem/cerebellum (range 0-3) & $3.0(3.0-3.0)$ & $0(0-3.0)$ & $<0.001$ \\
\hline
\end{tabular}

ASPECTS Alberta Stroke Program Early Computed Tomography Score

${ }^{a}$ ASPECTS-b; ASPECTS-b is extended to both sides from the original ASPECTS protocol

${ }^{b}$ MASPECTS, modified ASPECT; similar to ASPECTS-b (bilateral ASPECTS), each middle cerebral artery territory had 0-10 points. Anterior cerebral artery and posterior cerebral artery territories had 2 points, corresponding to upper and lower levels of each territory. The brainstem and each cerebellar hemisphere had 1 point 

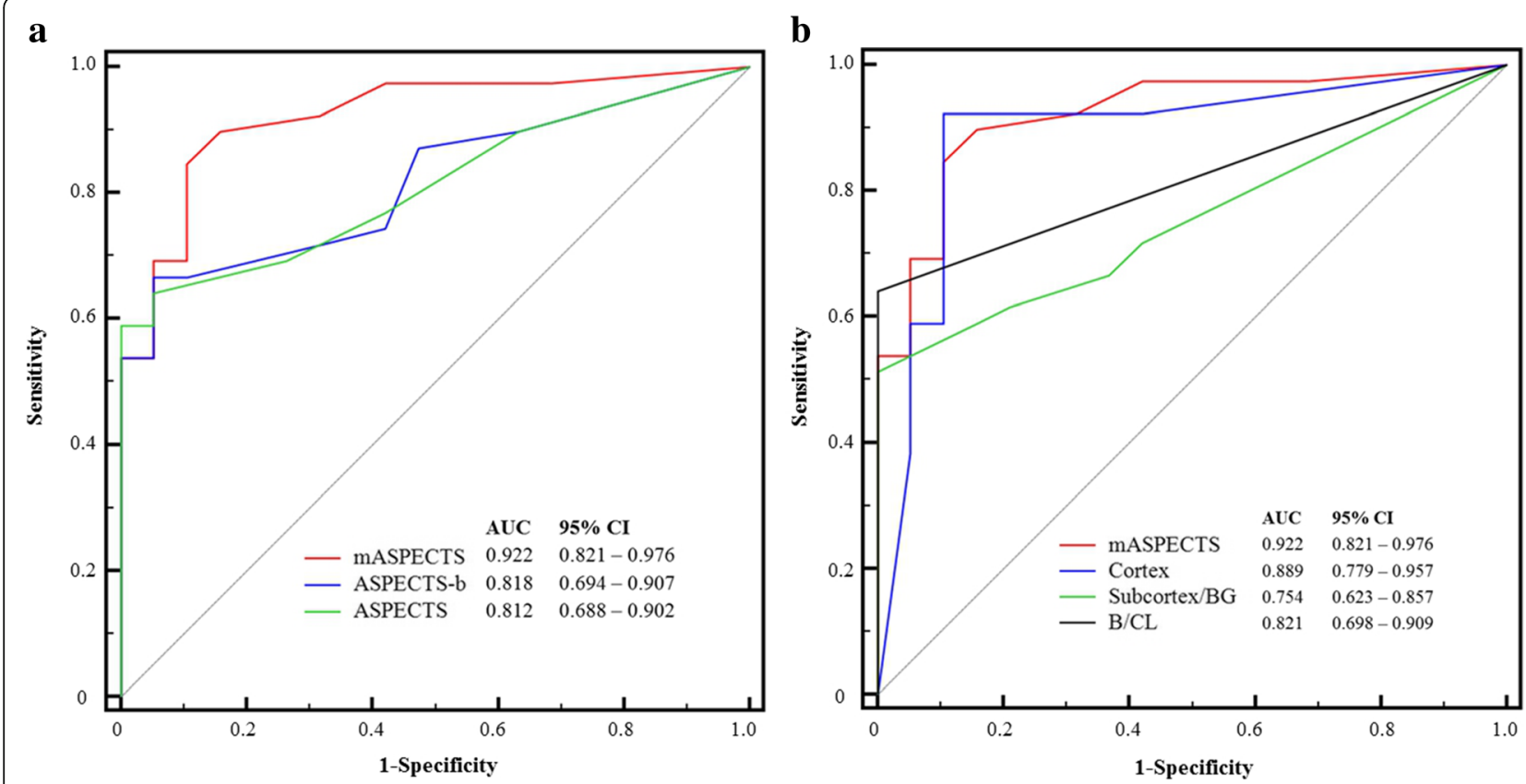

Fig. 3 ROC curves for the prediction of poor neurological outcome using ASPECTS, ASPECTS-b, and mASPECTS (a), as well as three regions of mASPECTS (b). ASPECTS Alberta Stroke Program Early Computed Tomography Score, ASPECTS-b Bilateral ASPECTS, mASPECTS Modified ASPECTS, BG Basal ganglia, B Brainstem, CL Cerebellum

neurological outcomes in patients after ECPR. Major findings of this study are as follows:

1. Brain CT could be helpful for predicting neurological outcomes of post-cardiac arrest patients after ECPR.

2. ASPECTS and its modifications were feasible and reliable for predicting neurological outcomes of post-cardiac arrest patients after ECPR.

3. The predictive performance of MASPECTS for poor neurological outcome was better than that of ASPECTS or ASPECTS-b.

4. In multivariable analysis, ischemic cardiac cause of arrest and mASPECTS were significant predictors of poor neurologic outcomes in ECPR patients.

Neuroimaging studies such as brain CT, brain magnetic resonance imaging, and magnetic resonance spectroscopy after conventional CPR have been performed [1]. Brain CT scans might be particularly helpful for predicting neurological outcomes after ECPR. However, brain magnetic resonance imaging is an expensive and time-consuming procedure. It cannot be performed immediately, because ECMO equipment is used for patients receiving ECPR [22]. In addition, sedative medications and neuromuscular blockade are commonly used in comatose CPR survivors. They might confound outcome prediction after cardiac arrest [23]. Some clinical signs and examinations such as motor response to noxious stimuli, corneal reflex, caloric testing, and EEG might also be confounded by sedation $[1,23]$. Therefore, brain CT might be more helpful for predicting neurological outcome in ECPR patients under sedation. However, data on prognostic markers of brain $\mathrm{CT}$ for neurological outcomes after ECPR are limited [17, 24].

Some changes visualized by brain CT have been associated with hypoxic-ischemic cerebral insult. They are useful for predicting poor neurological outcome after cardiac arrest [5-7, 25]. Especially, loss of the boundary between gray matter and white matter and cortical sulcal effacement on brain CT are associated with hypoxic-ischemic brain damage and poor outcome after cardiac arrest $[5-7,25]$. These changes are also useful for predicting poor neurological outcomes after ECPR [7]. However, identification of these signs might depend on the abilities of each investigator. In addition, these changes are not quantifiable. Therefore, an objective and quantifiable tool is needed to identify hypoxic-ischemic lesions. ASPECTS is a widely used screening tool to quantify the extent of ischemic hypodensity or hypoattenuation in ischemic stroke. ASPECTS and its modifications might be able to explain the quantitative association between cerebral insult following hypoxic-ischemic injury after cardiac arrest and neurological outcome $[9,10]$. In conventional CPR, ASPECT with some modifications could be used to estimate early neurologic outcomes in post-cardiac arrest patients $[6,9,10]$. 
Selective vulnerability has been reported for hypoxic-ischemic injury in previous studies. The middle lamina of the cortex, caudate nucleus, putamen, globus pallidus, thalami, and Purkinje cells might be at particular risk owing to highly metabolically active tissue [26, 27]. Eventually, cortex, basal ganglia, and the cerebellum are areas vulnerable to hypoxic-ischemic injury after cardiac arrest. In the present study, the performance of MASPECTS for predicting poor neurological outcome was better than ASPECTS-b. Although ASPECTS-b can estimate hypoxic-ischemic lesions in bilateral MCA regions (MCA territorial cortex, subcortex, and basal ganglia), mASPECTS can estimate lesions in bilateral regions of ACA, PCA, and the cerebellum as well as MCA regions of ASPECTS-b. Therefore, mASPECTS can better detect cortical lesions by hypoxic-ischemic insult than ASPECTS-b. In addition, cortical lesions seem to be more relevant to neurological outcomes after ECPR than those of subcortex/basal ganglia or the brainstem/cerebellum. These findings suggest that mASPECTS is more useful than ASPECTS or ASPECT-b for predicting neurological outcomes in patients after ECPR.

This study has several limitations. First, the CPC score was retrospectively determined on the basis of medical records. Second, the nonrandomized nature of the registry data might have resulted in selection bias. Although brain CT was performed within 48 hours following ECPR, a major limitation of this study might be that the CT was performed at different time settings. However, in ROC curves for the prediction of poor neurological outcome using CT scores, AUCs of CT scores were not significantly different between within and after median CT scan intervals in this study. Third, brain CT scan was performed by two different scanners, which might have resulted in bias. Fourth, selection bias might have been an influence because we excluded 26 non-survived patients for whom we could not exactly define neurological status at discharge. We added the result including these 26 patients to compare with the results excluding these patients in Additional file 2. Last, a limited number of patients received targeted temperature management. The methods used were decided by each intensivist. They varied according to conditions and situations.

\section{Conclusions}

In this study, ASPECTS with modifications was found to be feasible and reliable for predicting neurological outcomes in ECPR survivors.

\section{Key messages}

- Brain CT can be helpful for predicting neurological outcomes of post-cardiac arrest patients after ECPR.
- ASPECTS and its modifications were feasible and reliable for prediction of neurological outcomes of post-cardiac arrest patients after ECPR.

- The predictive performance of mASPECTS for poor neurological outcome was better than that of ASPECTS or bASPECTS.

\section{Additional files}

\author{
Additional file 1: Table S1. The findings of portable \\ electroencephalography (EEG) within 7 days after extracorporeal \\ cardiopulmonary resuscitation. (DOC $29 \mathrm{~kb}$ )
}

Additional file 2: Figure S1. mASPECTS according to neurological outcome. Bar is presented to median with interquartile range. There was a significant difference in $\mathrm{MASPECTS}$ according to neurological outcome $(p<0.001)$, and it was assessed by the Kruskal-Wallis test among groups. Bars of the same color indicate nonsignificant differences between groups based on the Mann-Whitney $U$ test. (DOCX $172 \mathrm{~kb}$ )

\section{Abbreviations}

ACA: Anterior cerebral artery; ASPECTS: Alberta Stroke Program Early Computed Tomography Score; ASPECTS-b: Bilateral ASPECTS; CPC: Cerebral Performance Categories scale; CPR: Cardiopulmonary resuscitation; $C T$ : Computed tomography; ECMO: Extracorporeal membrane oxygenation; ECPR: Extracorporeal cardiopulmonary resuscitation;

EEG: Electroencephalography; HUMC: Hallym University Medical Center; ICC: Intraclass correlation coefficient; ICU : Intensive care unit; mASPECTS: Modified ASPECTS; MCA: Middle cerebral artery; PCA: Posterior cerebral artery; ROSC: Return of spontaneous circulation; SMC: Samsung Medical Center

\section{Acknowledgements}

We thank Keumhee C. Carriere, PhD, and Joonghyun Ahn, MS, at the Samsung Biomedical Research Institute for their excellent statistical support.

\section{Funding}

The authors received no funding from any source for this research or the writing of this article.

\section{Availability of data and materials}

The data reported in this article is available via the Harvard Dataverse Network (https://doi.org/10.7910/DVN/SMFMTH) as a recommended repository of Critical Care.

\section{Authors' contributions}

JAR participated in study design, data collection, drafting of the manuscript, and the statistical analysis. YHL participated in study design, data collection, drafting of the manuscript, and the statistical analysis. CRC participated in the statistical analysis and helped to draft the manuscript. YHC participated in study design, performed the statistical analysis, and drafted the manuscript. KS participated in the statistical analysis and helped to draft the manuscript. KJ participated in study design and coordination. GYS participated in study design and coordination and helped to draft the manuscript. TKP participated in study design and drafting of the manuscript. JML participated in the statistical analysis and helped to draft the manuscript. MKC participated in study conception and design, data collection, and drafting of the manuscript. JHH participated in study design and coordination. SHL participated in the statistical analysis and helped to draft the manuscript. HSK participated in the statistical analysis and helped to draft the manuscript. JHY participated in study conception and design, data collection, and drafting of the manuscript. All authors read and approved the final manuscript.

\section{Ethics approval and consent to participate}

This was a retrospective, multicenter, observational study of adult patients who underwent ECPR during hospitalization at Hallym University Medical Center (HUMC) and Samsung Medical Center (SMC) between May 2010 and December 
2016. This study was approved by the institutional review board of HUMC (HUMC 2015i128) and SMC (SMC 2017-11-088-002). The requirement for informed consent was waived due to the study's retrospective nature.

\section{Consent for publication}

Not applicable. This study did not contain any individual person's data in any form (including individual details, images or videos).

\section{Competing interests}

The authors declare that they have no competing interests.

\section{Publisher's Note}

Springer Nature remains neutral with regard to jurisdictional claims in published maps and institutional affiliations.

\section{Author details \\ ${ }^{1}$ Department of Critical Care Medicine, Samsung Medical Center, Sungkyunkwan University School of Medicine, 81 Irwon-ro, Gangnam-gu, Seoul 06351, Republic of Korea. ${ }^{2}$ Department of Neurosurgery, Samsung Medical Center, Sungkyunkwan University School of Medicine, Seoul, South Korea. ${ }^{3}$ Department of Emergency Medicine, Soonchunhyang University Bucheon Hospital, Bucheon, South Korea. ${ }^{4}$ Department of Thoracic and Cardiovascular Surgery, Samsung Medical Center, Sungkyunkwan University School of Medicine, Seoul, Republic of Korea. ${ }^{5}$ Division of Pulmonary and Critical Care Medicine, Department of Medicine, Samsung Medical Center, Sungkyunkwan University School of Medicine, Seoul, South Korea. ${ }^{6}$ Division of Cardiology, Department of Medicine, Samsung Medical Center, Sungkyunkwan University School of Medicine, Seoul, Republic of Korea. 'Department of Emergency Medicine, Ajou University School of Medicine, Suwon, South Korea. ${ }^{8}$ Department of Neurology, Keimyung University Dongsan Medical Center, Daegu, South Korea. 'Department of Emergency Medicine, Hallym University Medical Center, Anyang, South Korea. \\ ${ }^{10}$ Department of Thoracic and Cardiovascular Surgery, Hallym University Medical Center, Anyang, South Korea.}

Received: 2 January 2018 Accepted: 19 June 2018

Published online: 22 November 2018

\section{References}

1. Young GB. Neurologic prognosis after cardiac arrest. N Engl J Med. 2009; 361:605-11.

2. Sandroni C, Cariou A, Cavallaro F, Cronberg T, Friberg H, Hoedemaekers C, et al. Prognostication in comatose survivors of cardiac arrest: an advisory statement from the European Resuscitation Council and the European Society of Intensive Care Medicine. Intensive Care Med. 2014;40:1816-31.

3. Naples R, Ellison E, Brady WJ. Cranial computed tomography in the resuscitated patient with cardiac arrest. Am J Emerg Med. 2009;27:63-7.

4. Hirsch KG, Mlynash M, Jansen S, Persoon S, Eyngorn I, Krasnokutsky MV, et al. Prognostic value of a qualitative brain MRI scoring system after cardiac arrest. J Neuroimaging. 2015;25:430-7.

5. Inamasu J, Miyatake S, Nakatsukasa M, Koh H, Yagami T. Loss of gray-white matter discrimination as an early $C T$ sign of brain ischemia/hypoxia in victims of asphyxial cardiac arrest. Emerg Radiol. 2011;18:295-8.

6. Chakraborty S, Symons SP, Chapman M, Aviv RI, Fox AJ. Diffuse ischemia in noncontrast computed tomography predicts outcome in patients in intensive care unit. Can Assoc Radiol J. 2012;63:129-34.

7. Ryu JA, Chung CR, Cho YH, Sung K, Suh GY, Park TK, et al. The association of findings on brain computed tomography with neurologic outcomes following extracorporeal cardiopulmonary resuscitation. Crit Care. 2017:21:15.

8. Barber PA, Demchuk AM, Zhang J, Buchan AM. Validity and reliability of a quantitative computed tomography score in predicting outcome of hyperacute stroke before thrombolytic therapy. Lancet. 2000;355:1670-4.

9. Sugimori H, Kanna T, Yamashita K, Kuwashiro T, Yoshiura T, Zaitsu A, et al. Early findings on brain computed tomography and the prognosis of postcardiac arrest syndrome: application of the score for stroke patients. Resuscitation. 2012;83:848-54

10. Lee KS, Lee SE, Choi JY, Gho YR, Chae MK, Park EJ, et al. Useful computed tomography score for estimation of early neurologic outcome in post-cardiac arrest patients with therapeutic hypothermia. Circ J. 2017;81:1628-35.

11. Chen YS, Lin JW, Yu HY, Ko WJ, Jerng JS, Chang WT, et al. Cardiopulmonary resuscitation with assisted extracorporeal life-support versus conventional cardiopulmonary resuscitation in adults with in-hospital cardiac arrest: an observational study and propensity analysis. Lancet. 2008;372:554-61.

12. Park SB, Yang JH, Park TK, Cho YH, Sung K, Chung CR, et al. Developing a risk prediction model for survival to discharge in cardiac arrest patients who undergo extracorporeal membrane oxygenation. Int J Cardiol. 2014;177:1031-5.

13. Ryu JA, Park TK, Chung CR, Cho YH, Sung K, Suh GY, et al. Association between body temperature patterns and neurological outcomes after extracorporeal cardiopulmonary resuscitation. PLoS One. 2017;12:e0170711.

14. Kang MJ, Lee TR, Shin TG, Sim MS, Jo IJ, Song KJ, et al. Survival and neurologic outcomes of out-of-hospital cardiac arrest patients who were transferred after return of spontaneous circulation for integrated postcardiac arrest syndrome care: the another feasibility of the cardiac arrest center. J Korean Med Sci. 2014;29:1301-7.

15. Cummins RO, Chamberlain DA, Abramson NS, Allen M, Baskett PJ, Becker L, et al. Recommended guidelines for uniform reporting of data from out-ofhospital cardiac arrest: the Utstein style. A statement for health professionals from a task force of the American Heart Association, the European Resuscitation Council, the Heart and Stroke Foundation of Canada, and the Australian Resuscitation Council. Circulation. 1991;84:960-75.

16. Cummins RO, Chamberlain D, Hazinski MF, Nadkarni V, Kloeck W, Kramer E, et al. Recommended guidelines for reviewing, reporting, and conducting research on in-hospital resuscitation: the in-hospital 'Utstein style'. A statement for healthcare professionals from the American Heart Association, the European Resuscitation Council, the Heart and Stroke Foundation of Canada, the Australian Resuscitation Council, and the Resuscitation Councils of Southern Africa. Circulation. 1997:95:2213-39.

17. Ryu JA, Cho YH, Sung K, Choi SH, Yang JH, Choi JH, et al. Predictors of neurological outcomes after successful extracorporeal cardiopulmonary resuscitation. BMC Anesthesiol. 2015;15:26

18. DeLong ER, DeLong DM, Clarke-Pearson DL. Comparing the areas under two or more correlated receiver operating characteristic curves: a nonparametric approach. Biometrics. 1988;44:837-45.

19. Schisterman EF, Perkins NJ, Liu A, Bondell H. Optimal cut-point and its corresponding Youden index to discriminate individuals using pooled blood samples. Epidemiology. 2005;16:73-81.

20. Ruopp MD, Perkins NJ, Whitcomb BW, Schisterman EF. Youden index and optimal cut-point estimated from observations affected by a lower limit of detection. Biom J. 2008;50:419-30.

21. Koo TK, Li MYA. Guideline of selecting and reporting intraclass correlation coefficients for reliability research. J Chiropr Med. 2016;15:155-63.

22. Lee YH, Oh YT, Ahn HC, Kim HS, Han SJ, Lee JJ, et al. The prognostic value of the grey-to-white matter ratio in cardiac arrest patients treated with extracorporeal membrane oxygenation. Resuscitation. 2016;99:50-5.

23. Samaniego EA, Mlynash M, Caulfield AF, Eyngorn I, Wijman CA. Sedation confounds outcome prediction in cardiac arrest survivors treated with hypothermia. Neurocrit Care. 2011;15:113-9.

24. Sakamoto T, Morimura N, Nagao K, Asai Y, Yokota H, Nara S, et al. Extracorporeal cardiopulmonary resuscitation versus conventional cardiopulmonary resuscitation in adults with out-of-hospital cardiac arrest: a prospective observational study. Resuscitation. 2014;85:762-8.

25. Torbey MT, Selim M, Knorr J, Bigelow C, Recht L. Quantitative analysis of the loss of distinction between gray and white matter in comatose patients after cardiac arrest. Stroke. 2000;31:2163-7.

26. Wijdicks EF, Campeau NG. Miller GM. MR imaging in comatose survivors of cardiac resuscitation. AJNR Am J Neuroradiol. 2001;22:1561-5.

27. Sekhon MS, Ainslie PN, Griesdale DE. Clinical pathophysiology of hypoxic ischemic brain injury after cardiac arrest: a "two-hit" model. Crit Care. 2017;21:90. 